\title{
High power microwave antenna design using infrared imaging techniques
}

\author{
by NORGARD J.*, SADLER J. ${ }^{\circ}$, BACA E. ${ }^{\circ}$, PRATHER W. ${ }^{\circ}$ SEGA R. ${ }^{+}$and SEIFERT R. **
}

"US Air Force Academy \& University of Colorado, Colorado Springs, CO 80933-7150, 'USAF, Phillips Laboratory, PLWSR, Kirtland AFB, NM 87117-5776 ${ }^{+}$NASA/JSC, Houston, TX 77058, *USAF Rome Laboratory, RLERST, Griffiss AFB, NY 13441-4505

Abstract

An infrared (IR) imaging technique is used to design a high power microwave (HPM) antenna. IR thermograms of the electric field distributions of the internal modes in several cross sections of the waveguide feed line to the antenna and the radiation patterns of a $30^{\circ}$ beveled-cut circular waveguide microwave aperture antenna are measured. A thin, lossy detector screen is placed in the plane of measurement. The absorbed heat energy in the screen is re-radiated as electromagnetic (EM) "blackbody" energy, which is detected with an IR camera. Due to the absorbed energy, the temperature of the detector screen rises above the ambient temperature of the waveguide by an amount proportional to the local electric field intensity (energy) at each point in the screen material. The temperature distribution in the screen material is correlated to the intensity of the electric field absorbed in the screen and is presented as a false-color image of the electric field distribution.

The antenna is fed with a coaxial $\mathrm{TM}_{01}$ mode, which does not radiate in the bore-sight direction of an open-ended cylindrical waveguide; therefore, a combined mode converted/radiator is used to convert the $\mathrm{TM}_{01}$ mode into the dominant circular waveguide $\mathrm{TE}_{11}$ mode, which does have a main beam radiation pattern. A $30^{\circ}$ beveled-cut radiator is used for mode conversion. The far-field radiation pattern of the beveled-end aperture antenna is also measured.

\section{Introduction}

An infrared (IR) imaging technique has been developed to map two-dimensional electric field distributions near an emitter or a scattering body. This technique is applied to design a highly directive HPM aperture antenna. The antenna is made from circular waveguide. The antenna is fed with a circularly symmetric coaxial $\mathrm{TM}_{01}$ mode. From symmetry, the $\mathrm{TM}_{01}$ mode does not radiate in the bore-sight direction from an open-ended circular waveguide; however, the dominant circular $\mathrm{TE}_{11}$ mode does have a directive main beam radiation pattern. Therefore, a mode converter is used to convert the symmetric $\mathrm{TM}_{01}$ mode into the dominant $\mathrm{TE}_{11}$ mode. To achieve the required mode conversion, the open-ended aperture of the circular waveguide is cut into a $30^{\circ}$ beveled-cut aperture (a conic section with an elliptical crosssection). This design is compact and efficient since it combines the mode converter with the directional radiator.

The far-field radiation pattern of the beveled-cut microwave aperture antenna is measured. Also, experimentally determined IR thermograms (field images) of the circular waveguide modes generated inside the waveguide are made in several cross-sectional planes along the feed line from the coaxial source to the elliptical aperture. These IR thermograms are compared with the theoretically predicted $\mathrm{CW}$ results in the interior of the cylinder in several cross-sectional planes in the feed line to the aperture. The field in the beveled aperture is of special interest and is also mapped with the IR imaging technique. The mode conversion process inside the waveguide from the source to the beveled aperture is determined in this manner.

\section{IR Imaging of electric fields}

An infrared (IR) measurement technique [1-6] has been developed to observe electric fields. This measurement technique produces a two-dimensional IR thermogram (image) of the The colour plates of this article are on pages I - $N$ at the end of the book. 
electric field being measured, i.e. a two-dimensional isothermal contour map or a gray scale of the intensity of the electric field.

The IR measurement involves the observation of microwave energy deposited in an electrically lossy detection screen placed in the plane over which the electric field is to be measured. Joule heating of the detector material results in IR emissions which can be detected by an IR imaging system. The detection screen material is calibrated such that a given temperature rise (above the ambient temperature of the material) corresponds to a particular electric field intensity level.

\subsection{Electrical parameters}

The detector screen is made from a thin sheet of linear, homogenous and isotropic lossy material. From the complex form of Poynting's Theorem for a linear, homogeneous and isotropic material, the absorbed power $P_{a b s}$ within a given volume $\mathbf{V}$ of the lossy material is a function of the electric $(E)$ and magnetic $(H)$ field intensities inside the screen and is given by

$$
P_{a b s}=\int_{V}\left(\sigma E^{2}+\omega \varepsilon^{\prime \prime} E^{2}+\omega \mu^{\prime \prime} H^{2}\right) d V\left[W / m^{2}\right]
$$

where $\sigma$ is the conductivity of the detector screen, $\varepsilon^{\prime \prime}$ is the imaginary component of the permittivity of the detector screen, $\mu^{\prime \prime}$ is the imaginary component of the permeability of the detector screen, and $\omega$ is the radian frequency of the incident field. The volume integral is over the illuminated portion of the detector screen.

The screen material is tailored to respond to only one component of the field. For example, the electric field detector screen used in this paper was constructed from a thin, planar sheet of lossy carbon impregnated film (Teledeltos Paper) with a high conductivity $\sigma$ and a low imaginary permittivity $\varepsilon$ " and a low imaginary permeability $\mu$ ". The detector screen is $80 \mu \mathrm{m}$ thick and has a conductivity of 8 mhos per meter. For this conducting, non-polarizable, non-magnetic screen material, maximum heating occurs due to the electric field, and negligible heating occurs due to the magnetic field.

The incident EM energy is absorbed by the lossy material and is converted into thermal heat energy which causes the temperature of the detector material to rise above the ambient temperature of the surrounding background environment by an amount which is proportional to the local electric field intensity (energy) at each point (pixel) in the screen material. In regions where the fields are strong, the absorbed energy is large and the resulting pixel temperatures are high; in regions where the fields are weak, the absorbed energy is small and the resulting pixel temperatures are low. The resulting two-dimensional temperature distribution over the surface of the screen is detected, digitized and stored in the memory of the IR camera. The temperature distribution on the surface of the screen without any EM energy incident on the screen is also stored in the memory of the IR camera as an ambient background reference temperature distribution. The difference in the temperature distributions at each pixel between the illuminated and the non-illuminated screens is due to the effects of the electric field incident on the screen at that pixel location. These EM effects can be visualized by presenting the differenced two-dimensional temperature profile as a false color image, where cool colors (for example shades of blue) represent weak areas of EM energy and hot colors (for example shades of red) represent strong areas of EM energy. The resulting two-dimensional false color image is called an IR thermogram, i.e. an iso-temperature contour map, and is a representation of the electric field distribution passing through the screen material.

\subsection{Thermal parameters}

For a planar sheet of detector screen supported by a block of non-conducting material, e.g. a styrofoam block, the thermal problem reduces to considering only the radiative and convective heat losses from the surface of the detector material.

The convective heat loss $h_{c}$ is approximated by

$$
h_{c}=h_{o}\left(T-T_{a m b}\right)^{1.25}\left[\mathrm{~W} / \mathrm{m}^{2}\right]
$$

where $h_{0}$ varies between 1.4 and 1.6. The radiative heat loss $h_{r}$ is approximated by

$$
h_{r}=\varepsilon_{i r} \sigma_{i r}\left(T^{4}-T_{a m b}^{4}\right)\left[W / m^{2}\right]
$$


http://dx.doi.org/10.21611/qirt.1994.004

where $\varepsilon_{i r}$ is the detector surface emissivity, $\sigma_{\mathrm{ir}}$ is the Stefan-Boltzman constant in $\mathrm{W} / \mathrm{m}^{2}-\mathrm{K}^{4}$, and the temperatures are in degrees Kelvin. The conductive heat loss is negligibly small.

\subsection{Thermal equilibrium}

The heat transfer problem in the detector material involves solving a non-linear, second order differential equation in both space and time, while considering radiative and convective heat losses from the surface of the material, conductive heat transfer within the material, and the EM power absorbed in the material as a function of distance into the material. For the case of the thin screens considered here, the temperature is initially considered to be constant in the direction normal to the surface of the material, so that the conductive term normal to the surface of the screen can be ignored and the power absorbed can be considered independent of the direction normal to the surface of the screen. Also, the time dependence of the absorbed heat energy is ignored for the steady-state solution.

Relating the convective and radiative heat losses in equations (2) and (3) to the absorbed power in equation (1), results in the following equation at thermal equilibrium:

$$
P_{a b s}=h_{c}+h_{r}
$$

Non-linear thermal/electrical equation can be solved for the electric or magnetic field, as a function of the material temperature $\mathrm{T}$, using approximate techniques.

\subsection{Approximate solution}

Equation (4) is highly non-linear for large temperature variations above ambient, due to the thermal processes of convection and radiation. However, for small temperature variations of only a few degrees above ambient, equation (4) can be linearized for small incremental temperature changes $\Delta T=T-T_{a m b}$ above the ambient temperature $T_{\text {amb }}$. For this minimally perturbing measurement case, an almost direct linear correlation exists between the incremental surface temperature $\Delta T$ and the absorbed electric field intensity. Therefore, it is possible to correlate local surface temperature variations $\Delta T$ to $E$ field intensities.

\subsection{IR thermograms (images)}

The temperature difference between the screen material and the background is detected, digitized, and stored in the memory of an IR camera on a pixel by pixel basis. The starring Focal Plane Array (FPA) system used to take the IR thermograms in this paper has 256 by 256 pixels per frame of data. Each pixel is an Indium Antimonide (InSb) IR photo detector operated in a photo voltaic mode. The array operates at liquid Nitrogen temperatures and is enclosed in a Dewar. The camera can detect temperature differences of approximately 0.009 ${ }^{\circ} \mathrm{K}$, and has a relative accuracy of plus or minus $10 \%$ when detecting electric field's.

Stored IR thermogram data represents the temperature distribution over the extent of the detector screen and is a map of the intensity of the electric field distribution absorbed in the screen.

\section{Experimental setup}

The IR imaging technique is applied to determine the modal distributions of the electric field excited inside a circular waveguide microwave aperture antenna. The modal structure of the electric field is measured in several cross-sectional planes inside the waveguide. A small, thin circular disk of carbon paper is placed inside the circular waveguide and positioned in the cross-sectional planes over which the electric field modes are measured. IR thermograms are made of the cylindrical modes excited inside the cavity in these planes. The brightness of each color in the image corresponds to the intensity of the EM field.

\section{Circular waveguide mode converter}

The radiator consist of a circular waveguide driving a radiating aperture, as shown in figure 1. The waveguide is fed on the source end with a coaxial probe centered on the axis of the waveguide, which produces a circularly symmetric $\mathrm{TM}_{01}$ coaxial mode. The aperture is formed 
on the radiating end of the waveguide by terminating the waveguide in a $30^{\circ}$ bevel cut, as shown in figure 1b. A standard open-ended circular waveguide aperture is shown in figure $1 a$.

\subsection{Open-end circular aperture}

The modal patterns of the electric fields at the cross-sections marked 1 through 4 in figure $1 a$ for the open-ended circular aperture are measured using a carbon disk positioned at these cross-sections. IR thermograms measured at positions 1 through 4 are all similar, since no mode conversion occurs at the open-ended aperture. The thermogram of the $T M_{01}$ mode shown in figure 2 (at position 1) is typical and representative of all other positions (1a, 2, 3, and 4 ) in the open-ended circular waveguide. Due to the circular symmetry of the coaxial TM01 mode, the $T M_{01}$ mode at the source end of the waveguide is not converted into the $T_{11}$ mode at the aperture end of the waveguide. The radiation pattern of the open-ended waveguide, as shown in figure 4, does not have a main beam in the direction of the axis of the waveguide.

The radiation pattern is a conical beam (first side lobe). This circular aperture antenna, therefore, has a very low gain.

\section{2. $30^{\circ}$ beveled-end elliptical aperture}

The modal patterns of the electric fields at the cross-sections marked 1 through 4 in figure $1 \mathrm{~b}$ for the $30^{\circ}$ beveled-ended elliptical aperture are also measured using a carbon disk positioned at these cross-sections. IR thermograms at positions 1 through 4 are different, as shown in figure 3, because mode conversion occurs in the beveled aperture. The IR thermograms shown in figure 3 (at position 1a) again show the circularly symmetric $T_{01}$ mode, identical to the modes at the equivalent positions in the open-ended waveguide. However, the IR thermograms shown in figure 3 (at positions 2 and position 3) show the TM $_{01}$ mode mixed with a dominant $T_{11}$ mode. The IR thermograms shown in figure 3 (at position 4 - in the aperture) show the dominant $T_{11}$ mode, created due to the mode conversion of the $T M_{01}$ mode to a $T E_{11}$ mode in the beveled aperture. The $T E_{11}$ mode generated in the beveled section is reflected off the end of the aperture back to positions 2 and 3 . Notice that the movement of the areas of constructive interference shown in figure 3 is due to phase changes inside the waveguide between positions 2 and 3 . The radiation pattern of the beveled-ended aperture does have a main beam, as shown in figure 5 . It is, however, refracted toward the normal of the aperture away from the axis of the waveguide and, therefore, is not aligned in the direction of the axis of the waveguide, as shown in figure 6 . This aperture antenna, nevertheless, has a very high gain, in the off-axis direction of the main beam, as shown in figure 7a. At $14 \mathrm{GHz}$, the directivity of the antenna is approximately $13 \mathrm{~dB}$. The corresponding half-power beam width of the antenna is approximately 28 degrees.

\section{3. $30^{\circ}$ shaped-end elliptical aperture}

The modal patterns of the electric fields with in the $30^{\circ}$ beveled-end waveguide also show higher order modes present within the waveguide. The double lobes in figure 3 (at $d=3.75 "$ and at $\left.d=3.5^{\prime \prime}\right)$ are due to the $T E_{21}$ mode mixing with dominant $T E_{11}$ mode. The triple lobes in figure 3 (between $d=3.25$ " and $d=2 "$ ) are due to the $T E_{31}$ mode mixing with the dominant $T E_{11}$ mode. To reduce the generation and reflection back into the waveguide of these undesirable modes the edges of the $30^{\circ}$ beveled-edge was shaped into a smooth contour. The radiation pattern of the shaped beveled-ended aperture has a higher gain, as shown in figure 7 . At 18 $\mathrm{GHz}$, the directivity of the antenna is approximately $19 \mathrm{~dB}$. The corresponding half-power beam width of the antenna is approximately 18 degrees.

\section{Conclusions}

The IR thermograms presented in this paper show the various stages of mode conversion in the combined mode converter/radiator with a $30^{\circ}$ beveled-ended elliptical aperture (with and without shaping) from the coaxial low gain circularly symmetric $\mathrm{TM}_{01}$ mode at the source end of the waveguide to the high gain dominant circular $T E_{11}$ mode at the aperture end of the waveguide. 
http://dx.doi.org/10.21611/qirt.1994.004

Determining which modes were produced and at what positions these modes occurred inside the waveguide was helpful in the overall design of the mode converter and helped confirm and verify the final design procedures. Comparisons between the theoretical predictions and the measured IR results were made to verify the accuracy of the theoretical predictions.

This example illustrates the use of the IR technique to correlate theoretical data with experimental observations and to experimentally aid in the design of high gain microwave antennas.

\section{REFERENCES}

[1] METZGER (D.W.), NORGARD (J.D.) and SEGA (R.M.) "Near-Fields Patterns from Pyramidal Horn Antennas: Numerical Calculation \& Experimental Verification" IEEE-EMC Transactions, Vol. 33, No. 3, August 1991, pp. 188-196.

[2] SEGA (R.M.) and NORGARD (J.D.), "Infrared Measurement of Scattering and Electromagnetic Penetrations through Apertures," IEEE-NS Transactions, Vol. NS-33, No.6, December 1986, pp. 1658-1661.

[3] NORGARD (J.D.), SEGA (R.M.), IANACONE (K.J.), HARRISON (M.G.), PESTA (A.T.) and SEIFERT (M.A.), "Scattering Effects of Electric and Magnetic Field Probes," IEEE-NS Transactions, Vol. NS-36, No. 6, December 1989, pp. 2050-2057.

[4] SEGA (R.M.) and NORGARD (J.D.), "An Infrared Measurement Technique for the Assessment of Electromagnetic Coupling," IEEE-NS Transactions, Vol. 32, No. 6, December 1985, pp. 4330-4332.

[5].SEGA (R.M.), NORGARD (J.D.) and GENELLO (G.J.), "Measured Internal Coupled Electromagnetic Fields Related to Cavity and Aperture Resonance," IEEE-NS Transactions, Vol. NS-34, No. 6, December 1987, pp. 1502-1507.

[6] NORGARD (J.D.) FROMME (D.C) and SEGA (R.M.) "Correlation of Infrared Measurement Results of Coupled Fields in Long Cylinders with a Dual Series Solution," IEEE-NS Transactions, Vol. NS-37, No. 6, December 1990, pp. 2138-2143.

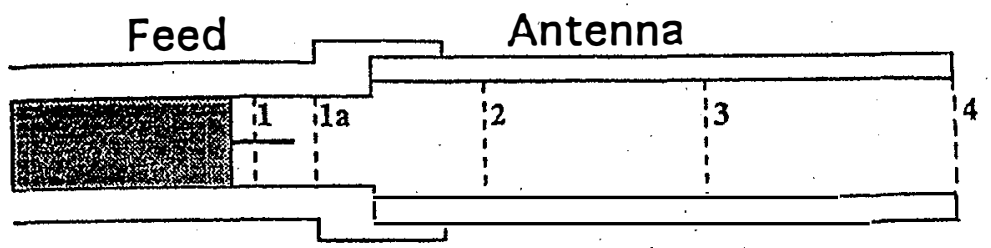

a. Open-end circularwaveguide aperture antenna

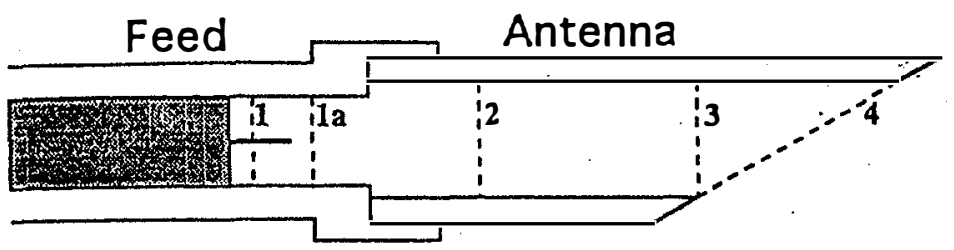

b. $30^{\circ}$ beveled-end elliptical aperture antenna

Fig. 1. - Experimental setup for IR images of the internal modes in a cylindrical waveguide microwave aperture antenna 